\title{
Activité dialogique et micro-improvisations en entretien de conseil en orientation
}

Dialogical activity and micro-improvisations in the career counseling interview

\section{Isabelle Olry-Louis}

\section{(2) OpenEdition}

\section{Journals}

Édition électronique

URL : http://journals.openedition.org/activites/973

DOI : 10.4000 /activites. 973

ISSN : $1765-2723$

\section{Éditeur}

ARPACT - Association Recherches et Pratiques sur les ACTivités

\section{Référence électronique}

Isabelle Olry-Louis, « Activité dialogique et micro-improvisations en entretien de conseil en orientation », Activités [En ligne], 12-1 | Avril 2015, mis en ligne le 15 avril 2015, consulté le 21 avril 2019. URL: http://journals.openedition.org/activites/973; DOI : 10.4000/activites.973

\section{(9) $\odot \Theta \Theta$}

Activités est mis à disposition selon les termes de la licence Creative Commons Attribution - Pas d'Utilisation Commerciale - Pas de Modification 4.0 International. 


\title{
Activité dialogique et micro-improvisations en entretien de conseil en orientation
}

\author{
Isabelle Olry-Louis
}

Université Paris Ouest Nanterre, LAPPS /TE20, Laboratoire Parisien de Psychologie sociale, EA 4386,
Équipe Travail, Ergonomie, Orientation \& Organisations - iolry@u-paris10.fr

ABSTRACT

Dialogical activity and micro-improvisations in the career counseling interview. We advocate the utility of considering the ordinary interactions of career counseling as an activity which needs to be analysed in reference to theories of the activity, to situated action and to conversational analysis. Our position may be summarized as follows: 1) counselling is always improvised to some extent (micro-improvisations); 2) this has to be the case, due to the properties of the situation, namely the asymmetry of the relationship between the consultant and the counsellor, the former being asked to provide the "materials" upon which the latter interactionally performs his/her professional acts; 3 ) so whilst they both have to improvise, they do so in different ways, because their respective uncertainties have different sources: the consultant never knows exactly what he/she is supposed to say in a given situation and the counsellor never knows what effects his/her interventions will have; 4) the success of a vocational counselling interview therefore depends on the degree of adjustment the partners will be able to reach in a joint process seen as a "dialogical improvisation". We will then focus on the vocational counselling activity as a joint process in which takes place during the course of situated interactions, with the objective of gaining a better understanding of the pragmatic processes involved in the monitoring of the interaction progress. Empirical data will be taken into account through the analysis of a certain number of excerpts from a corpus of interviews carried out by young vocational counsellors.

\section{KEYWORDS}

activity, conversational analysis, dialogical interactions, career counseling interview, improvisation

Les entretiens de conseil en orientation et, plus largement, les entretiens menés dans le cadre de la gestion de carrière ou de l'insertion font aujourd'hui l'objet de prescriptions multiples dont la formulation oscille entre recommandations générales et préconisations précises pouvant prendre la forme de scripts conversationnels. Certaines de ces prescriptions, définies dans des cadres légaux et souvent spécifiées en contexte institutionnel, portent sur le format et la place de l'entretien dans des prestations individuelles comme le bilan de compétences, le parcours d'orientation professionnelle individuel et la validation des acquis de l'expérience (Delory-Momberger, 2007; Lacaille, 2012 ; Mayen, 2009). D'autres sont inscrites dans des réflexions épistémo-philosophico-morales propres à l'orientation et portent sur sa finalité, ses objets et son déroulement idéal (Guichard, 2008 ; Savickas, 2011).

Constatant que l'entretien constitue, et de loin, la méthode la plus utilisée dans le champ de l'orientation au point de faire figurer les métiers du conseil vocationnel parmi ceux qui s'accomplissent essentiellement au moyen de la parole, il nous apparaît utile de chercher à 
mieux connaître la façon dont s'effectuent les interactions ordinaires de conseil. Aussi, plutôt que de définir a priori «des formes normatives d'entretiens » optons-nous pour une voie délibérément empirique visant à décrire les interactions de conseil telles qu'elles s'accomplissent dans le cours de l'action. Nous considérons en effet urgent de chercher à mieux saisir les processus dialogiques du conseil auxquels dans ce domaine, les trop rares appels à l'utilisation de méthodes qualitatives (Bernaud, 2012; Hanson, Creswell, Plano Clark, Petsk, \& Creswell, 2005 ; Schilling, 2006) et les plus rares encore références explicites à l'analyse de l'activité n'ont pour le moment pas permis de donner un éclairage suffisant (Olry-Louis, 2013).

En quoi les interactions de conseil se distinguent-elles d'autres interactions ? À quelles pratiques discursives se livrent conseiller et bénéficiaire de l'entretien ? Quels processus langagiers sous-tendent ces jeux de dialogue? Avec quels effets conversationnels et psychologiques ? Chercher à répondre à ces questions suppose selon nous de poser préalablement un cadre théorique et méthodologique propice à la spécification de l'entretien de conseil, à l'examen des processus langagiers qu'il mobilise et à la caractérisation de l'action qui s'y accomplit. Après que ces points auront été développés dans la section suivante, nous pourrons aborder la problématique de l'improvisation pour l'entretien de conseil et formuler les hypothèses inhérentes à ces questions dans les deux sections ultérieures. La quatrième section sera consacrée à l'exposé de la méthode permettant d'appréhender l'activité discursive des conseillers et consultants. Le détail de notre analyse de discours sera présenté dans une cinquième section.

\section{1.- De l'entretien de conseil conçu comme genre à celui défini en tant qu'activité}

De façon générale, l'entretien peut être défini comme un évènement de parole («speech event ») dans lequel une personne A extrait une information biographique d'une personne B (Labov \& Fanshel, 1977). Dans le cas où B a l'initiative de la rencontre et/ou en bénéficie directement, l'entretien est une consultation qui prend la forme d'un dialogue finalisé entre deux personnes aux rôles complémentaires (François, 1993 ; Vion, 1992). Disposant d'un savoir socialement reconnu, un professionnel se trouve interpellé par un consultant qui prend l'initiative de l'échange dans l'attente d'un bénéfice escompté pour lui-même.

\section{1.- Caractéristiques génériques de l'entretien clinique et de l'entretien de conseil}

Parmi les consultations professionnelles, l'entretien clinique a pour spécificité de chercher à « comprendre le fonctionnement psychologique d'un sujet en se centrant sur son vécu et en mettant l'accent sur la relation » (Bénony \& Chahraoui, 1999, p. 11). Il se distingue d'autres entretiens professionnels du fait de l'attention portée par le praticien au point de vue de son interlocuteur, qui l'amène à lier son propre discours au sien de façon peu directive (Grossen \& Salazar Orvig, 2006). En dépit de la variabilité des pratiques auxquelles il peut donner lieu, plusieurs de ses traits caractéristiques ont été soulignés (Grossen \& Trognon, 2002). À côté du fait que le contexte institutionnel dans lequel il prend place exerce de nombreuses contraintes sur l'interaction et que son déroulement se conforme à des formats d'intervention psychologique, on retiendra surtout que l'entretien clinique est marqué par une forte asymétrie de compétences entre les interlocuteurs, ne serait-ce que dans la connaissance de la situation d'interaction et des actions attendues de chacun. Les rôles complémentaires pris par le psychologue et son client leur confèrent en effet des positions distinctes par rapport à la parole : le premier est supposé savoir susciter la parole de son interlocuteur pour en «faire quelque chose » tandis qu'il appartient au second de fournir un discours permettant au premier d'accomplir interactionnellement ses gestes professionnels. On retiendra aussi que le contenu évoqué par les interactants peut, par son caractère très personnel, rompre avec certaines règles de politesse (discrétion, évitement de thèmes délicats). 
Dans le champ de l'orientation et de l'insertion, c'est l'entretien de conseil qui apparaît le plus souvent mobilisé, aux côtés de l'entretien de recherche et de celui de sélection professionnelle (Guichard \& Huteau, 2007). C'est par son objectif propre que le premier se différencie «en ce qu'il se déroule dans l'intérêt de l'interviewé (à son bénéfice), alors que le deuxième l'est primordialement au bénéfice du chercheur (et du développement des savoirs) et que le troisième l'est d'abord au profit de l'entreprise qui recrute (et secondairement du candidat) » (ibid., p.174). Par ailleurs, la visée clinique revendiquée dans l'entretien de conseil non directif centré sur le client, tel qu'il a été défini par Rogers, s'accommode assez mal de celle essentiellement descriptive de l'entretien de recherche ou de celle fondamentalement évaluative de l'entretien de recrutement. Ce dernier rend en effet les enjeux identitaires des interactants explicites et substantiels, "la mise en scène d'une image positive de soi étant contractuellement requise, et la production discursive de l'évalué y est manifestement guidée par un modèle supposé doté de valeur sociale » (Camus, 2004, p. 158).

L'entretien de conseil constitue donc un type particulier d'entretien clinique avec une structure thématique orientée préférentiellement vers des objets tels que le passé scolaire et professionnel, la situation et la trajectoire familiale, l'état du projet, les obstacles rencontrés, etc. Prenant toujours place dans un dispositif socio-institutionnel qui en contraint le format, ce dialogue finalisé vise à «aider une personne à trouver la voie professionnelle et de (ou de) formation et, plus généralement, la forme de vie qui lui convient le mieux et à s'engager dans la direction qu'elle aura ainsi déterminée » (Guichard \& Huteau, ibid, p. 174). Cet entretien est conçu pour faire éprouver des expériences de transformation/reconstruction de soi, d'où la forme généralement peu directive qui lui est donnée. C'est la formulation par le consultant d'une matière propice à l'élaboration d'un projet ou, plus largement, de ce qui fait problème dans l'orientation qui est recherchée par le psychologue. La dimension dialogique de l'entretien de conseil est fondamentale puisque «le sens ne se situe ni dans le locuteur, $n i$ dans l'auditeur: il se trouve dans l'interaction entre les deux partenaires. Dans le dialogique, le sens est co-travaillé »(Lhotellier, 2000, p. 32).

La caractérisation a priori du «genre » dont relève l'entretien de conseil en orientation, de sa fonction et des processus sur lesquels il repose, tout comme la prescription de formes normatives d'entretiens de conseil en orientation sont relativement fréquentes dans la littérature (voir notamment Egan, 1994 ; Gysbers, Heppner, \& Johnson, 1998; Manthei, 1997 ; Rogers, 1951). Plus rares et marginaux sont les travaux empiriques qui décrivent le déroulement effectif des tours de parole au cours d'entretiens réels d'orientation ayant fait l'objet d'enregistrements (Doublet, 2006 ; Zarka, 2000). Alors que ces dernières années, d'autres entretiens professionnels, notamment l'entretien thérapeutique (Labov \& Fanshel, ibid.) et divers entretiens institutionnels prélevés dans le champ social (Drew \& Heritage, 1992), ont été objets d'études visant à en observer le déroulement au sein de situations concrètes, l'observation d'entretiens de conseil en orientation effectifs visant à décrire l'activité discursive qui s'y déploie et à formuler des hypothèses sur les processus sousjacents reste à accomplir.

Une telle perspective nécessite selon nous d'inscrire la recherche dans une filiation théorique se référant explicitement aux théories de l'activité et de l'action située ainsi qu'à l'analyse de discours. Pour ce faire, il nous faut reprendre brièvement quelques éléments notionnels qui se trouvent à la croisée de ces domaines en soutenant l'idée qu'ils pourraient bien constituer les fondements d'une approche pragmatique des situations dialogiques de conseil.

\section{2.- Concepts pour penser l'entretien comme une activité}

Ancrées en psychologie du développement et inspirée par l'école russe (Leontiev, 1974 ; Vygotski, 1997), les théories de l'activité, qui se sont déployées dans plusieurs champs parfois au détriment de leur unité, partagent cependant entre elles le fait de mettre l'accent 
sur le sujet. Elles conçoivent les transformations du sujet comme prenant racine dans le développement d'activités extérieures et socialisées. Entre le sujet de l'activité et l'objet visé par celle-ci, des médiations symboliques et artefactuelles s'accompliraient qui, en s'intériorisant, mèneraient à une double dynamique de développement : celle du sujet et celle de l'activité. En somme, loin de considérer un sujet donné à l'avance, les théories de l'activité conçoivent de multiples reconfigurations des fonctions psychologiques et de la conscience à travers les actions exercées en situation.

Le courant de l'action située, inscrit dans la continuité des perspectives ethnométhodologiques, insiste lui sur la situation. Si le plan conçu par le sujet est une ressource car il donne une orientation à son action, ce n'est pas lui qui rend compte de l'action effective. Cette dernière émerge des circonstances. Plus précisément, «l'organisation de l'action est entendue comme un système émergent in situ de la dynamique des interactions » (Conein \& Jacopin, p. 476) et il convient alors d'étudier comment les gens utilisent les circonstances pour effectuer une action intelligente (Suchman, 1987). À travers sa réflexivité et son indexicalité, la communication verbale constitue alors le prototype d'une approche contextuelle de l'action, l'énonciation permettant l'ancrage dans la situation (Béguin \& Clot, 2005). On retrouve ici l'héritage des approches interactionnistes pour lesquelles les individus, lorsqu'ils sont en présence physique immédiate les uns des autres, contribuent à définir ensemble la situation dans laquelle ils se trouvent (Goffman, 1973) et concourent, par leurs interactions, à modifier un ordre social qui, loin d'être donné objectivement, résulte d'une improvisation réglée (Blumer, 1969 ; Mead, 1934). Au niveau microscopique d'une situation dialogique particulière, c'est au gré de la dynamique intersubjective qu'ils parviendraient à créer de nouveaux contextes pour l'action (Grossen, 2001).

$\mathrm{Si}$ ces approches constituent pour Licoppe (2008) deux des grandes orientations ethnographiques bordant l'espace de recherche centré sur le travail, leur confrontation met au jour certaines tensions. Non seulement elles adoptent des postures différentes sur la dialectique du changement et de la continuité, abordant chacune à leur manière les liens entre le donné et le créé ou entre le fonctionnement et le développement (Béguin \& Clot, ibid.), mais elles engendrent également des différences importantes dans la manière même d'observer l'activité. Pour la théorie de l'activité, une grande part des données empiriques est constituée de verbalisations sur l'accomplissement de l'action portées par les agents euxmêmes : qu'elles soient le fruit d'entretiens d'explicitation ou entretiens d'auto-confrontation (simple ou croisée), ces données sollicitent la subjectivité des agents qui participent activement à l'élaboration et à l'interprétation des données. Pour les théories de l'action située, de telles données sont problématiques en raison de leur caractère rétrospectif. On leur préfère des méthodes qui s'inscrivent dans le cours d'action et permettent de saisir empiriquement la manière dont les personnes s'orientent dans les micro-situations rencontrées. C'est dans ce cadre que l'analyse de la conversation est convoquée, sur la base des travaux de Sacks (1992) et de Schegloff (1987) qui étudient l'organisation séquentielle des conversations ordinaires, professionnelles ou institutionnelles entendues ici comme des séquences d'actions.

Rapportée à la situation d'entretien de conseil, la perspective théorique privilégiée engendre, on le voit, des conséquences méthodologiques importantes. Sans dénier le primat de l'intentionnalité des personnes, de leur capacité d'agir et de se développer, nous avons pour notre part choisi de minimiser le poids de la sollicitation de processus conscients d'interprétation de l'action. Notre analyse se base essentiellement sur les procédures discursives attestant de la façon dont les personnes engagées dans la situation construisent et font reconnaître son intelligibilité au fil de l'action dialogique en train de se faire. Tout au long de l'entretien, moment après moment, on s'intéresse donc aux processus langagiers d'énonciation, de construction de la situation et de négociation du sens mobilisés conjointement par le consultant et par le conseiller. Ce sont les tournants de l'activité 
discursive qui orientent tout particulièrement notre attention. Selon nous, ils ne sauraient procéder de la simple application de plans et de règles qui seraient fixés à l'avance par les interlocuteurs, pas plus qu'ils ne résulteraient des seules circonstances. Dans le cadre de cette activité conjointe qu'est le «tenir conseil » (Lhotellier, ibid.), laquelle s'élabore dans l'ici-et-le-maintenant d'une interaction vive, nous les voyons plutôt comme le fruit de tentatives d'ouverture initiées par l'un des partenaires auxquelles l'autre ajuste sa propre action, certaines séquences s'apparentant à une véritable improvisation dialoguée comme nous allons l'exposer à présent (Olry-Louis \& Grillo, 2014).

\section{2.- Place de l'improvisation dans l'entretien de conseil}

\section{1.- L'improvisation : une inscription dans l'action}

Improvisation et action entretiennent de puissants liens d'interdépendance: toute improvisation s'inscrit dans un cours d'action et aucune action ne saurait être à l'abri d'un imprévu venant contraindre sa forme. Improvisent ainsi au travail celles et ceux qui aménagent le prescrit pour pouvoir agir efficacement dans les organisations, ces ajustements devant être presque à chaque fois réinventés (Dejours, 2009). Improvisent aussi celles et ceux qui rencontrent des moments critiques dans le déroulement des actions qu'ils sont engagés à accomplir. C'est le cas lorsqu'une rupture affecte le cours de l'action initiée au point de venir brusquement imposer de procéder autrement; l'improvisation est alors une tentative pour poursuivre l'action jusqu'à ce que soit restaurée une continuité, au moins relative, avec l'ordre antérieur ou habituel des choses.

Lorsqu'on n'est pas seul à improviser face à une situation, mais que celle-ci implique un alter avec lequel il faut composer, la notion devient pragmatique : l'action de l'un peut surprendre l'autre et le mettre en difficulté, elle peut aussi amener les actants à accomplir ensemble quelque chose de singulier voire d'original, eu égard aux normes en cours. Aussi convient-il selon nous d'entendre l'improvisation selon deux manières : la première, fondée sur la notion d'urgence, concerne l'adaptabilité à une situation inattendue ou de crise, elle requiert de mobiliser les ressources les plus ingénieuses pour y faire face et vise au retour d'un état stable ; la seconde se veut en rupture avec un ordre établi. S'opérant souvent au mépris des genres habituels, bien qu'elle puisse prendre appui sur la maîtrise des codes et des règles, elle vise à produire quelque chose d'inédit. Dans les deux cas, nous retiendrons que l'improvisation consiste à rompre avec une certaine continuité et à approcher le danger, sollicitant la mobilisation judicieuse et rapide de ressources déjà présentes. Mais, plus encore que son mode d'accomplissement - sans préparation minutieuse ni chemin attendu, sans protocole fixe, stratégie clairement définie ni esprit de système - c'est son contexte d'apparition (imprévu) qui la caractérise, l'inattendu ne concernant pas nécessairement l'évènement qui se produit, mais le moment auquel il se produit, difficile à anticiper (Popelard, 2014). De façon générale, cet imprévu ne saurait être radical, il est toujours relatif à un sujet concret, situé dans un contexte d'action et souvent pris dans des rapports sociaux.

Les théories de l'action située accordent une grande importance à l'improvisation. En effet pour elles, l'action est moins considérée comme le produit de la compétence des acteurs que comme une habileté à s'ajuster aux circonstances et à les utiliser en saisissant les occasions au moment favorable, ce qui requiert d'improviser sans cesse (Livingstone, 2008 ; Ogien \& Quéré, 2005). Ainsi les agents seraient-ils toujours en alerte sur les suites à donner à leur action, à tout moment focalisés sur l'appréciation de l'état de la situation. Corps, émotions, perceptions, raisonnement pratique, constitueraient alors autant de ressources mobilisables contribuant aux ajustements afin de corriger, réorienter ou reconfigurer les conduites dès que la situation le requiert. Dans un cadre dialogique, chacun des interlocuteurs, attentif par un jeu d'anticipations et de rétrospections, à ce que la parole (la sienne propre et celle du partenaire) produit, règlerait au fur et à mesure ses interventions sur les précédents actes 
langagiers en prenant en compte leurs effets. Et cet ajustement réciproque devrait être attesté au niveau même de la structure du dialogue.

Pour ces théories, on peut dire que c'est à mi-chemin entre le hasard et la routine que l'improvisation prend place: une action entièrement livrée au hasard n'aurait que peu de chances d'être ordonnée et ne laisserait guère de possibilité de contrôle à l'agent ; une action purement routinière serait quant à elle dépourvue de raisonnement, puisque les enchaînements s'opèreraient de façon purement mécanique. C'est donc là où une conduite non mécanique manifeste une cohérence, une forme, un ajustement que l'improvisation préside à l'ordonnancement d'un cours d'action (Ogien \& Quéré, ibid.).

En somme, l'improvisation peut être décrite comme une action qui se déploie dans l'instant, sans anticipation, en réponse à une situation imprévue. Parce qu'elle contraint à s'écarter des voies habituelles, elle est périlleuse et fait appel à de nombreuses ressources disponibles chez le sujet et dans son environnement. Marquée par son contexte d'apparition, celui d'une rupture avec ce qui précède et par la forme structurée qu'elle est supposée prendre pour s'avérer opérante, elle nécessite des ajustements entre les partenaires dès lors qu'elle s'inscrit dans une situation dialogique. Ainsi définie, l'improvisation trouve-t-elle place dans l'entretien de conseil en orientation?

\section{2.- Les micro-improvisations conversationnelles du conseil en orientation}

$\mathrm{Si}$, conformément à un principe de sérialité des énoncés, chaque acte langagier est supposé découler d'un autre l'ayant précédé dans l'entretien, et ouvrir la voie à ceux qui le suivent, le «point d'arrivée » étant fonction de tout ce qui l'aurait à chaque fois précédé, on peut s'interroger sur les logiques non mécaniques présidant à l'ordonnancement effectif des tours de parole. Car l'entretien de conseil présente généralement une forme peu directive dont le psychologue attend, comme c'est le cas pour tous les entretiens cliniques, quelle l'aide à comprendre le point de vue du consultant, à coordonner ses propres contributions aux siennes et à établir peu à peu avec lui une relation de confiance (Grossen \& Trognon, ibid.). Dans de tels entretiens, la situation dialogique est fréquemment redéfinie en fonction des évènements qui se produisent au cours de la rencontre et des effets produits par les actes langagiers. En cela, c'est une rencontre qui comporte un grand nombre d'inconnues et se révèle, à ce titre, non dénuée de risques (Blanchet \& Gotman, 2006).

Aussi, bien qu'elle s'inscrive dans un cadre institutionnel avec ses scripts, ses formats et ses routines, l'action discursive propre à l'entretien de conseil est d'abord à envisager comme une dynamique qui se développe dans la temporalité d'une activité conjointe constamment incertaine. En effet, si d'un point de vue formel, tout contenu conversationnel se présente comme une suite d'énoncés temporellement ordonnés, distribués entre les partenaires et organisés en diverses architectures, ils n'en sont pas moins partiellement imprévisibles (Trognon, 1999, 2002). Pour ses actants, la question se pose à chaque moment de savoir comment poursuivre l'action initiée en fonction à la fois de ce qu'il en comprend dans l'instant et de ce qu'il en attend. S'ajuster aux dires de l'interlocuteur, saisir les occasions favorables dans le fil de son discours, faire face aux évènements susceptibles de surgir dans l'interaction, en utilisant au mieux les circonstances, tout cela requiert d'improviser.

Comme l'action est précisément de nature conversationnelle, c'est-à-dire constituée d'unités très fines - les tours de parole et au sein de chacun d'eux des actes de langage - qui caractérisent les interventions de chaque interlocuteur, il est plus juste de parler ici de microimprovisations. Celles-ci renvoient en effet explicitement à un grain d'analyse d'une grande finesse, qui permet d'examiner dans le détail la manière dont, alternativement, conseiller et consultant répondent à la parole de l'autre et de tester l'idée selon laquelle, si chacun des partenaires est bien amené à improviser, il ne le fait pas nécessairement de la même façon et /ou pour les mêmes raisons. 


\section{3.- Hypothèses}

Il nous faut ici revenir sur l'asymétrie dans laquelle tous deux se trouvent de fait presque d'entrée de jeu. D'un côté, le conseiller ne peut connaître à l'avance le «matériau» sur lequel il aura à travailler, mais il dispose de savoirs et savoir-faire conversationnels sur lesquels il peut prendre appui pour gérer l'imprévu. De l'autre côté, le consultant n'a pas nécessairement une vision claire de ce qu'on attend de lui ni même de ce qu'il peut en attendre, n'ayant pour seuls guides que les interventions du conseiller et un savoir très partiel de la situation (Chabrol, 2000). Partant de ce constat, on formulera les hypothèses suivantes :

Premièrement, on s'attend à ce que la dissymétrie de places se traduise par des ruptures et discontinuités majoritairement initiées par le consultant, plaçant alors le conseiller devant l'obligation de sortir momentanément de son rôle habituel - par exemple en opérant un recentrage sur l'objectif principal de l'entretien, ce qui suppose de sa part l'acceptation provisoire et improvisée d'un nouvel objectif - tout en assurant la poursuite de l'entretien afin de le ramener si possible à son cours normal. Il y aurait en effet potentiellement toujours une part d'aléatoire dans les interventions du consultant, dont le conseiller est bien obligé de tenir compte. Même si ses interventions entendent orienter les réponses du consultant, ce dernier ne livre jamais que ce qu'il est prêt à énoncer à ce moment précis du dialogue. Aussi ses interventions peuvent-elles s'avérer « décalées » par rapport aux demandes formulées par le conseiller, soit parce qu'elles n'en constituent que des réponses fragmentaires, soit parce qu'elles ouvrent, sans forcément établir un rapport explicite avec ce qui précède, un nouvel épisode consacré à un récit de soi qui peut prendre la forme d'une confidence ou d'une révélation (Olry-Louis, Brémond, \& Pouliot, 2012).

Deuxièmement, cette asymétrie fondamentale devrait en quelque sorte s'inscrire dans les discours : on attendrait plutôt de la part du consultant un discours saturé en incises, hésitations, bafouillages, phrases heurtées ou inachevées, et de la part du conseiller des interventions sobres et concises visant à questionner, cadrer, orienter, relancer, dans un souci manifeste de clarté. Pour autant, dès lors qu'un nouvel espace improvisé est ouvert par l'un, l'autre devrait alors en cas d'ajustement inscrire son discours au plus près de celui du partenaire, de sorte que les contrastes de positionnement énonciatif s'estompent à ce moment du dialogue.

Troisièmement, on peut s'attendre à de grandes différences interindividuelles dans la propension à s'ajuster des conseillers, certains d'entre eux se refusant à s'écarter des voies convenues, s'arc-boutant sur des modes d'intervention définis à l'avance, en dépit de leur caractère inapproprié à la tournure du dialogue, d'autres faisant preuve d'une plus grande souplesse adaptative, acceptant de suivre le consultant sur des chemins inattendus et périlleux. Dans tous les cas, cela devrait se trouver marqué dans la structure même de l'échange.

\section{4.- Méthode}

En prenant pour unités d'analyse les micro-improvisitions, définies comme marquant une rupture conversationnelle avec ce qui précède, au niveau thématique ou énonciatif, et en considérant que ces micro-improvisations peuvent se limiter à un énoncé, prendre la forme d'une argumentation ou d'un récit si elles s'étendent sur de plus longues séquences, ou constituer un événement communicatif si elles sont coextensives à l'interaction (KerbratOrecchioni, 2005), nous avons cherché à extraire d'un vaste corpus d'entretiens retranscrits plusieurs épisodes relevant de micro-improvisations dialoguées, que nous avons soumis à une analyse discursive détaillée, effectuée au niveau des tours de parole (Alvesson \& Karreman, 2000). 


\section{1.- Le corpus}

Le corpus est constitué par 64 entretiens de conseil en orientation en direction d'adultes ou de jeunes adultes reçus dans diverses structures - Centre de Bilans, Mission Locale... - par des psychologues de l'orientation en fin de formation. Variés dans leur forme, leur durée et leur finalité, ils relevaient la plupart du temps d'une première rencontre, parfois destinée à donner lieu à d'autres - comme dans le cas des bilans de compétences. Ces entretiens ont été menés par 64 jeunes praticiens distincts, encore peu expérimentés, mais engagés dans une démarche réflexive puisqu'ils les ont enregistrés et retranscrits afin de porter un regard critique sur leurs pratiques.

\section{2.- Les épisodes analysés}

C'est l'enchaînement de tours de parole (Sacks, Schegloff, \& Jefferson, 1974) qui rend décelable l'irruption de séquences consacrées à une improvisation dialoguée. Des marqueurs d'ouverture sont à rechercher dans la présence d'éléments signalant une rupture conversationnelle liée, soit au contenu - la micro-improvisation initiale devrait être marquée par un déplacement thématique -, soit au positionnement énonciatif - elle devrait être attestée par une modification du rapport établi à son propre discours.

Partant de l'idée que les micro-improvisations du consultant devraient souvent marquer la transition vers un récit de soi, on recherchera dans quelle mesure elles sont caractérisées par un registre thématique associé à l'intime et au «difficile à dire »(Pop, 2007). Ce discours pouvant être accompagné de traces d'affects, nous rechercherons l'emploi groupé de répétitions, de pauses et d'allongements, d'interjections, l'intensité intonative et énonciative ou la désorganisation syntaxique indiquant généralement l'émotion (Plantin, 2003). L'emploi de marques du moi et l'absence de marques de modulation signalent aussi que le discours est exclusivement autocentré. Pour qualifier la manière dont les conseillers ajustent leurs interventions aux micro-improvisations du consultant, on examinera si leur registre sémantique fait écho ou se situe en rupture avec celui du consultant au travers de l'usage des hétéro-reformulations, des marques de questionnement et de feed-back.

Comme l'entrée en séquence improvisée est censée s'accompagner de déplacements thématiques ou de changements de registre, le problème de leur validité au sein de l'espace discursif institutionnel établi risque de se poser. Ces déplacements peuvent être attendus dans le cadre des entretiens de conseil en orientation et contribuer à nourrir potentiellement l'objet de travail qui réunit les participants. Ils peuvent aussi constituer des transgressions par rapport aux règles conversationnelles de discrétion et d'évitement de thèmes délicats. Aussi s'efforcera-t-on de décrire la manière dont cette séquence est clôturée par les protagonistes, par le biais d'une nouvelle rupture conversationnelle, visant à réorienter l'entretien.

\section{3.- Les modalités de l'analyse de discours}

L'analyse des épisodes proposée entend porter non seulement sur le contenu des échanges ce qui est dit -, mais sur la manière dont ceux-ci s'accomplissent - comment c'est dit. Plutôt que chercher, comme cela a été effectué dans d'autres travaux sur des données assez proches (par exemple, Kostulski \& Prot, 2004), à décrire les transactions comme une organisation des échanges qui se tisse par le déploiement d'une logique réalisant les propriétés des actes de langage qui y sont accomplis, on a préféré se référer à une analyse de discours traitant de la place des interlocuteurs et de leurs relations dans les discours, comme y convoque le champ de l'énonciation plus propice, selon nous, au repérage et à la caractérisation des micro-improvisations que nous cherchons à saisir au fil du dialogue. Ayant ouvert de grandes possibilités de recherche en analyse de discours, le champ de l'énonciation s'intéresse plus particulièrement à la «mise en fonctionnement de la langue par un acte individuel d'utilisation» (Benveniste, 1974, II, p. 80), autrement dit par la façon dont la 
langue se réalise, lors d'un acte énonciatif individuel, en parole. Concevant le discours comme instrument fondamental de constitution du sujet, Benvéniste est le premier à avoir considéré que des marques linguistiques observables dans l'énoncé traduisent des mécanismes spécifiques à l'acte d'énonciation. Pour lui, la situation à laquelle un énoncé réfère et lui donne signification tient au lieu, au moment, aux interlocuteurs en présence et à leur inscription dans le discours. Aussi, les marqueurs linguistiques portant la présence du locuteur dans son énoncé et susceptibles de renseigner son rapport au monde sont à rechercher parmi les embrayeurs et/ou les déictiques au sens large, expressions renvoyant à un référent dont l'identification est à opérer nécessairement au moyen de l'entourage spatiotemporel de leur occurrence. Il désigne en particulier : 1) les indicateurs de personne (je, tu) qui, loin de renvoyer directement à un concept ou à un individu, permettent au locuteur de devenir sujet d'un discours adressé à un récepteur, face à une troisième personne désignant le référent dont on parle, 2) les indices d'ostension, c'est-à-dire les indicateurs organisant les relations spatiales et temporelles autour du sujet pris comme repère, 3) les temps verbaux (présent, imparfait...) étant entendu que c'est dans la langue que se manifeste l'expérience du temps, 4) les marques de modalisation désignant l'attitude du locuteur à l'égard de son propre énoncé.

\section{5.- Illustrations empiriques}

Pour étayer notre démonstration, nous avons choisi de nous focaliser sur des moments emblématiques de l'entretien de conseil, ceux au cours desquels nous observons des confidences, définies comme étant des tentatives de livrer intentionnellement quelque chose de soi-même présentant une certaine valeur et pouvant prendre la forme de véritables révélations (Olry-Louis \& Brémond, 2010 ; Olry-Louis, et al.). Ainsi avons-nous tout d'abord sélectionné dans le corpus initial trois entretiens marqués par une activité discursive consacrée à la confidence au sein desquels nous avons extrait plusieurs courts épisodes focalisés sur l'énonciation de l'un de ces moments clés. Ces extraits donnent à voir des usages contrastés de micro-improvisations, dont nous nous proposons de rendre compte d'un point de vue interactionnel et énonciatif. En nous centrant sur leur mode d'entrée, leur énonciation par le consultant et leur traitement par le conseiller, nous nous attacherons à identifier certaines formes de régularités énonciatives, sans masquer pour autant la singularité des épisodes décrits eu égard aux situations et aux pratiques individuelles.

Les deux premiers extraits sont issus d'un entretien non programmé avec Mme A., une consultante de 45 ans que la conseillère (notée C) accepte de recevoir dans le cadre d'une prestation de retour à l'emploi. L'extrait 1 donne à voir les tout premiers moments de l'échange.

Extrait 1 : Improviser pour trouver un terrain d'entente

$\mathrm{C} 1$ - Bonjour Mme A, je suis consultante stagiaire dans le cabinet $\mathrm{X}$ et je vais vous accompagner tout au long de cette prestation qui durera trois mois et où l'on se verra tous les quinze jours. Je vous écoute.

A1 - Bonjour... 1'ANPE m'a dit de venir...

$\mathrm{C} 2$ - Que vous ont-ils dit à notre sujet ?

A2 - Vous allez me donner du travail?

C3 - Ce n'est pas exactement cela, mon rôle de conseillère en insertion professionnelle est [...]

A3 - Donc, pas de travail non plus ici...

$\mathrm{C} 4$ - À vous entendre, je comprends que vous vous attendiez à trouver du travail dans notre cabinet ?

A4 - Oui, je suis découragée... je cherche depuis si longtemps... [...]

Tandis que la conseillère tente d'expliciter et de faire expliciter, par des questions ouvertes (C1 et $\mathrm{C} 2)$ le cadre d'une première rencontre qui survient, de fait, à l'improviste et met en jeu un tiers - ici l'ANPE présentée par Mme D. comme l'investigatrice de sa venue - la consultante répond par une autre question à travers laquelle elle met à l'épreuve une attente que sa démarche a nourrie (A2: «Vous allez me donner du travail ?»). Traduisant une 
vision peu claire de ce qu'elle peut en espérer, voire même une méconnaissance du comportement - y compris verbal - attendu d'elle, cette intervention témoigne de l'asymétrie des partenaires en ce début d'entretien, l'une parfaitement rompue aux codes de l'entretien et à ses modes d'ouverture habituels, l'autre ne sachant par quoi commencer, qui s'aventure alors dans la mise en mots d'hypothèses toutes personnelles somme toute peu réalistes. Les deux tours suivants prennent exactement la même forme : dans une assez longue intervention, non retranscrite intégralement ici, la conseillère reprend une explicitation portant sur son rôle et ses modalités d'intervention, à laquelle répond la formulation d'une conclusion, tirée comme pour elle-même par Mme A. (A3 : « Donc pas de travail ici non plus ».) D'un côté, le référent conversationnel est la situation d'entretien présente, avec ses objectifs et ses modalités propres, de l'autre côté, il est orienté autour du travail, objet de préoccupation et ultime finalité recherchée. C'est l'intervention suivante de la conseillère $(\mathrm{C} 4:$ : A vous entendre, je comprends que vous vous attendiez à trouver du travail dans notre cabinet ?») qui va leur ouvrir un terrain d'atteinte quant à l'objet de leur focalisation conjointe : le récit de son parcours par Mme A. On notera au passage combien, en énonçant ainsi sa relance, la conseillère s'ajuste à sa partenaire, tant sur le contenu (les attentes sur le travail) que sur la forme, à travers le choix d'une modalité interrogative et la désignation des énonciateurs («à vous entendre, je comprends»). Ainsi, aux deux microimprovisations de Mme A., qui cherchent moins à s'inscrire dans le dialogue en cours (une question en réponse à une question, puis une conclusion personnelle sans rapport explicite avec ce qui précède) qu'à verbaliser ce qui relève d'un dialogue intérieur au risque de sortir des attendus du cadre, la conseillère répond en les ré-injectant dans un cours d'entretien revenu ainsi «à la normale ».

L'épisode suivant (C37 à A44) survient un peu plus tard, après que Mme A., qui s'avère en grande difficulté tant professionnelle que personnelle et familiale, ait évoqué les problèmes comportementaux qu'elle rencontre dans son travail - violences physiques récurrentes à l'encontre de personnes avec lesquelles elle travaille - et alors que l'entretien s'oriente vers la recherche d'explications à cette agressivité. Outre ses propres difficultés personnelles une difficulté notamment à supporter le regard d'autrui - la consultante évoque à ce moment le contexte personnel et familial actuel très difficile dans lequel elle évolue, caractérisé notamment par le récent décès de sa mère.

Extrait 2 : Improviser autour d'une succession de révélations brutales

C37 - Si j'entends bien, vous constatez un changement en vous depuis la mort de votre maman?

Mme A. 37 -C'est sûr ! Je suis à bout, je suis plus énervée, je n'ai plus rien à perdre.

C38 - Plus rien à perdre...

Mme A. 38 - A part mon dernier fils, non je n'ai plus rien à perdre. Mes filles sont mieux sans moi, mon compagnon va me quitter et donc je reste juste pour le petit. D'ailleurs, le petit, ils veulent me l'enfermer! C39-Qu'est-ce que vous voulez dire?

Mme A. 39 - Le dernier, B., ils veulent le mettre à l'hôpital pour les fous ! C40 - Ils?

Mme A. 40 - Les médecins. L'école a alerté le médecin parce qu'apparemment mon fils se met en danger aussi pour ses petits camarades. Il a des «troubles du comportement» qu'ils disent ! Moi, je connais cet endroit, ils veulent le mettre à $Y$ (nom de l'institution). Moi je connais, j'y suis allée et c'est pas joli, joli, ce qu'ils font là-bas (s'emporte en disant cela, élève la voix, se lève).

C41 - Si vous le voulez bien, nous allons reprendre tranquillement ce que l'on disait juste avant, à savoir que vous étiez inquiète par rapport à votre fils.

Mme A. 41 - Oui, mon petit, ils vont lui faire du mal comme ils m'ont fait (silence prolongé).

C42 - Pourquoi les médecins lui feraient du mal, Madame A. ?

Mme A. 42 - Moi, j'ai eu des attouchements là-bas. [...] Je ne veux pas qu'on lui refasse des horreurs.

C43 - Qu'on lui refasse?

Mme A. 43 - Oui, il a déjà eu des petits soucis quand il était plus jeune.

C44-Ce que j'entends, Madame, c'est que vous n'avez pas vécu des choses très faciles.

Mme A. 44 - Oui, c'est vrai et puis aussi je garde tout pour moi. J'évite de raconter ma vie aux premiers venus. Je me méfie, je redoute qu'on se moque encore et toujours. 
L'extrait 2 s'ouvre avec une question (C37) visant à faire expliciter les transformations internes consécutives à la perte évoquée par Mme $\mathrm{A}$. La réponse fournie suscite une réitération en écho de la conseillère (C38 : «Plus rien à perdre...»). La convocation du dernier fils dans le récit, en tant que seul objet échappant à ce " plus rien », va introduire une rupture dans l'échange qui, dès lors, prend une tournure inattendue, marquée sous le sceau d'une révélation brutale (Mme A38: «D'ailleurs ils veulent me l'enfermer ») qui va structurer toute la suite de l'échange. Les deux questions qui s'ensuivent visent à faire expliciter cette assertion déroutante quant à l'intention de signifier (C39: «qu'est-ce que vous voulez dire?») puis à l'agentivité $(\mathrm{C} 40$ : «ils ?»). La réponse occasionne un développement relatif aux circonstances de cette prise de décision et surtout à son issue en termes de lieu d'internement (Mme A40). On notera, ce faisant, le passage de la troisième personne («les médecins », «l'école», «mon fils», « ses petits camarades », « ils») à la première personne («je connais» «j’y suis allée »). Ce déplacement s'accompagne de manifestations comportementales de Mme A. (elle s'échauffe, hausse la voix, se lève) qui non seulement témoignent de son implication émotionnelle, mais viennent aussi conforter les propos antérieurement tenus sur les actes de violence dont elle est capable. Face à cet emportement inopiné, la conseillère tente un recadrage (C41) visant à préserver la continuité thématique («reprendre ce que l'on disait juste avant») et comportementale («tranquillement») de l'entretien. Dans son énoncé, elle convoque alternativement son interlocutrice pour susciter son adhésion ( $\mathrm{Si}$ vous le voulez bien»), puis leur objet de travail commun («nous allons» «ce que l'on disait»), et enfin Mme A. vue non plus comme sujet adhérant à la tâche, mais comme susceptible de développer l'appréciation que lui inspire la situation de son fils («à savoir que vous étiez inquiète par rapport à votre fils»). Cela n'empêche pas celle-ci (A41) de poursuivre le récit de sa propre histoire évoquée au tour précédent, passant ainsi du «mon petit» à «ce qu'ils m'ont fait » et du futur au passé. La relance interrogative alors formulée par la conseillère (C42 : «Pourquoi les médecins lui feraient du mal Madame A ?») tente de la ramener vers un discours argumentatif au sujet du futur de son fils, soulignant l'incongruité de la supposition de Mme A. et prenant bien soin de re-convoquer les médecins plutôt que le «ils » qui précède. La réponse apportée par Mme $\mathrm{A}$. (A42) s'énonce à la première personne et se conjugue à nouveau au passé, lequel apparaît doublement susceptible de se répéter: les événements qu'elle a connus jadis risquent de toucher à présent son fils et ceux qui semblent avoir marqué celui-ci antérieurement risquent de se reproduire. La reprise en écho par la conseillère du nouvel élément qui vient d'être introduit (C43: «Qu'on lui refasse ?») suscite une réponse, directe, impliquant cette fois la même personne que celle faisant l'objet de la question (Mme A43 : «Oui, il a déjà eu...»). Tout ce qui pesait sur le discours de Mme A. dans son interaction avec la conseillère a alors été prononcé. Celle-ci clôt ce moment de récit au moyen d'une intervention qui, en présentant les événements relatés comme appartenant au passé et en les attribuant pleinement à Mme A. (C44: "Ce que j'entends Madame, c'est que vous n'avez pas vécu des choses très faciles »), instaure une rupture thématique et permet de replacer Mme A. dans son présent (A44).

Parce qu'il survient abruptement dans l'entretien, qu'il s'accompagne de manifestations émotionnelles et comportementales «déviantes» et potentiellement menaçantes, qu'il met en scène des épisodes violents et transgressifs, cet échange mobilise pleinement la capacité à faire face de la conseillère. Son attitude ouverte, finalement assez peu cadrante, la qualité de son écoute et sa propension à ajuster son énonciation au plus près de celle de la consultante permettent à celle-ci de faire entendre l'origine de sa souffrance, même si la première s'emploie dès lors à refermer cet espace conversationnel difficilement soutenable qu'elle a pourtant bien voulu laisser se déployer. Ni l'une ni l'autre n'ont nécessairement anticipé ce qui allait s'y dire, ni même ne l'ont souhaité, mais elles y ont contribué toutes deux en fabriquant à chaque tour de parole ce qui peu à peu en vient à constituer le fil directeur de ce qu'il convenait de percer à jour. 
On peut dire que ce premier entretien est traversé de bout en bout par l'improvisation, et ce, pour plusieurs raisons. Premièrement, n'ayant pas fait l'objet d'un rendez-vous préalable, il est effectué «au pied levé ». Deuxièmement, le malentendu auquel il donne lieu dans ses premiers instants («on ne me donnera pas de travail ici») atteste du fait qu'il n'a pas été entendu de la même manière par les interlocutrices, qui doivent donc composer avec le contrat de communication pour s'ajuster un tant soit peu l'une à l'autre sur un objet commun. Troisièmement, toute une série de révélations est produite par la consultante, qu'elle n'avait vraisemblablement pas l'intention de livrer au préalable (cf. sa remarque initiale) et auxquelles elle semble peu accoutumée («J'évite de raconter ma vie aux premiers venus »). Ces révélations qui formatent en quelque sorte la suite de l'échange ne vont pas sans produire de l'effet chez la conseillère, ce dont atteste sans ambigüité le compte-rendu qu'elle fait ultérieurement de son entretien lorsqu'elle y est invitée (à propos de l'intervention C44, elle dit d'elle-même : " je ne sais plus du tout quoi dire à Mme A. Elle vient de me raconter des évènements pénibles et je suis affectée. Je tente de dire que j'entends ce qu'elle me dit, je tente de la rassurer, mais je suis toute retournée moi-même »). La conseillère n'en laisse néanmoins rien paraître à ce moment. On notera cependant, à partir de là, plusieurs tentatives de sa part de cadrer plus fortement le propos de son interlocutrice et de s'acheminer vers la clôture de l'entretien (cf. six tours de parole plus loin C50 : « Donc, si je comprends bien, on part sur les éléments suivants : un travail de préférence dans des locaux et non chez des particuliers, dans la commune de préférence et ce travail ne doit pas nécessiter le port de charges trop lourdes. C'est cela ?»).

Les deux extraits suivants prennent place au cœur d'un premier entretien mené par une conseillère (appelée à nouveau $\mathrm{C}$ ) auprès d'un adulte que nous nommerons B., âgé de 26 ans, reçu pour la première fois dans le cadre dans le cadre du diagnostic orientation à destination des RMIstes. Titulaire d'une licence en sciences sociales et disposant d'une expérience dans le secteur socio-éducatif, il se demande s'il doit poursuivre dans cette voie, entreprendre un master ou concilier les deux. L'entretien porte sur le projet professionnel et sur les problèmes que celui-ci suscite ou réactive.

\section{Extrait 3 : improviser pour chercher à dire et à faire dire}

C24 - Vous avez l'impression que l'on ne peut être utile que dans le secteur que vous avez choisi ?

B24 - Non pas complètement... on peut se sentir utile en montant sa boîte et en embauchant des gens qui en ont besoin... dans le management... mais disons que j'ai envie de me sentir utile vraiment pour des gens qui sont dans le besoin... qui ont vraiment de grosses difficultés... les handicapés... peut-être aussi à cause de mon père je sais pas...

$\mathrm{C} 25$ - (blanc) De votre père?

B25 - (blanc, décrochage du regard et manipulation du stylo) Oui... souvent je le regarde... je sais pas... je ne trouve pas mes mots.

C26 - B., j'entends ce que vous me dites et si vous n'avez pas envie d'en parler, c'est votre choix et je le respecte.

B26 - (blanc, Y. manipule toujours son stylo, décrochage du regard pour expliquer ce qui suit) C'est pas que je n'ai pas envie d'en parler, mais c'est un peu difficile... avec mon père c'est un peu compliqué depuis toujours... comme il est en fauteuil roulant... alors de temps en temps il m'énerve aussi... je me suis dit que je ferais mieux au niveau des études... mais bon au final je me suis arrêté à la licence comme lui. $\mathrm{C} 27$ - Vous éprouvez le besoin de faire mieux que votre père ?

B27 - Oui, j'aimerais bien... mais j'ai comme un barrage... faut que j'arrive à me motiver... peut-être mieux... je sais pas

C28 - Il faut que vous arriviez à vous motiver ?

B28 - (blanc). Oui ...que je puisse faire quelque chose que j'aime aussi... si on n'est pas motivé, on n'arrive à rien et surtout on commence, mais rien ...j' ai quand même envie de vivre normalement... mais c'est vrai qu'en ce moment je me pose beaucoup de questions par rapport à mon avenir professionnel... [...]

Dans l'extrait 3, une transition s'accomplit au détour d'une réponse formulée par le consultant (B24: « ... mais disons que j'ai envie de me sentir utile vraiment pour des gens qui sont dans le besoin... qui ont vraiment des grosses difficultés... les handicapés... peut-être 
aussi à cause de mon père je sais pas...»). Dans la même réplique, il passe du registre professionnel - celui du secteur dans lequel il se sent utile - au registre personnel, intime, de la relation au père, transition opérée par une série d'associations : se sentir utile / pour des gens qui ont de gosses difficultés / les handicapées / mon père. À la relance C25 (« de votre père? ») qui entérine la rupture en manifestant l'acceptation du changement de registre et donc le glissement du rôle assumé par la conseillère, le consultant poursuit (B25 : « ...oui...souvent je le regarde...je sais pas...je ne trouve pas mes mots ». La réplique de la conseillère $(\mathrm{C} 26:$ : B, j'entends ce que vous me dites et si vous n'avez pas envie d'en parler, c'est votre choix et je le respecte...») s'ajuste aux tentatives de révélation de soi énoncées par B., lui signifiant que, dans le sous-jeu qui s'inaugure, lui seul a l'initiative de l'orientation à donner à ses dires. Ainsi autorisé et mutuellement ratifié, l'échange se poursuit ainsi pendant quelques tours, nourri par les propositions hachées de B. qui tente d'énoncer, comme pour lui-même, sa relation au père (C27 à B28). Bien qu'éprouvant et particulièrement difficile, ce qu'attestent les nombreux décrochements de regard de B., ses hésitations et ses modalisations marquées par l'incertitude ( «je sais pas » réitéré plusieurs fois), cet échange semble avoir des effets positifs pour lui au vu de l'extrait 4 .

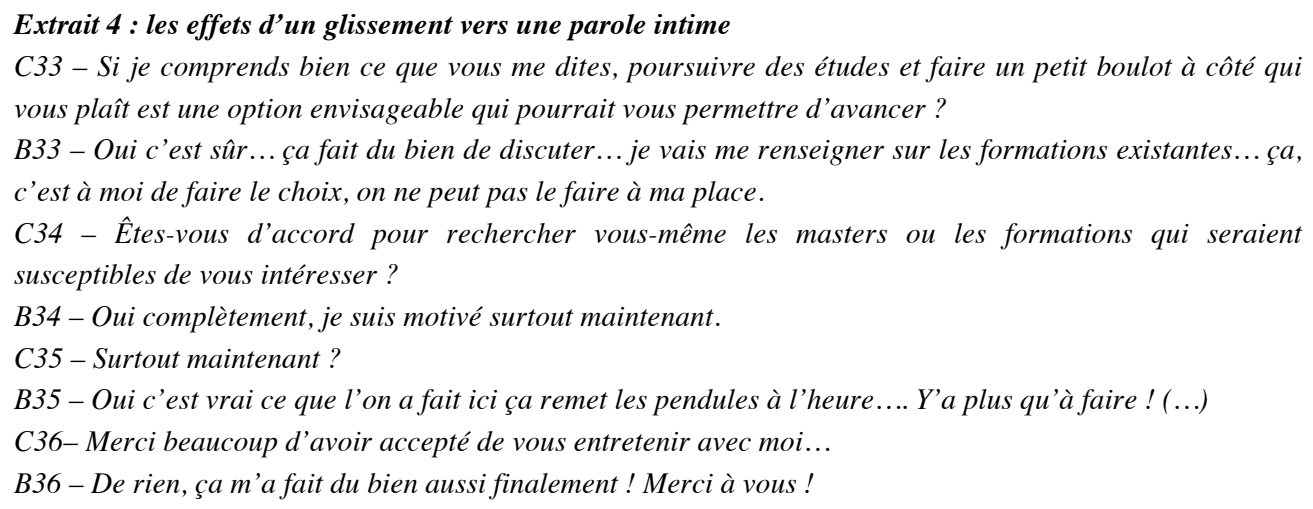

La parole qui cherche à se dire, stimulée à la fois par le climat de confiance initié par la conseillère et par la tension de B., est vraiment introduite par une «révélation » de B. au détour d'une intervention qui peut être qualifiée de micro-improvisation dans la mesure où rien des contenus abordés jusqu'alors n'avait permis de présager l'irruption de la thématique « handicap ». Dès lors, la conseillère le suit dans cette voie incertaine et hésitante, s'ajuste en le relançant à plusieurs reprises. Les énoncés restent inachevés, souvent maladroits, mais la rencontre semble avoir produit des effets sur B qui manifeste un changement d'état émotionnel palpable, bien qu'aucun dire définitif n'ait été énoncé (B33 : «ça fait du bien de discuter » puis B35: «Oui c'est vrai ce que l'on a fait ici ça remet les pendules à l'heure», puis encore B36 : « Ça m'a fait du bien aussi finalement !»).

Le cinquième extrait prend place au cours d'un entretien caractérisé par la brièveté des interventions des protagonistes, et par sa forme questions-réponses. Une jeune mère célibataire de 25 ans titulaire d'un baccalauréat technologique tertiaire, que nous appellerons D. rencontre pour la première fois, dans le cadre d'un dispositif d'insertion pour parents isolés, une conseillère (dite C.) qui fait porter l'entretien sur «les éléments pris en compte dans l'élaboration du projet professionnel ». Au $28^{\text {ème }}$ tour de parole, peu d'éléments ont été avancés pour nourrir l'entretien et faire progresser la compréhension du problème d'orientation de D. Prenant place dans le cadre d'actions de conseil en orientation destinées à de jeunes mères célibataires en difficulté, cet entretien s'est focalisé depuis le début sur le projet professionnel de la consultante et sur les éléments à prendre en compte pour l'élaborer, sans que la question de l'arrivée de la petite fille dans sa vie n'ait été abordée jusqu'alors. Il faut préciser, car le compte-rendu fait par la conseillère au sujet de sa propre activité insiste sur ce point, que l'entretien se déroule conformément à une succession de thèmes à aborder prévue dans le cadre d'un guide de questionnement que la conseillère s'est 
forgé préalablement et qu'elle s'efforce de suivre scrupuleusement, ce qui semble courtcircuiter les possibilités d'improvisation qui se présentent au fil de l'entretien.



L'extrait 5 donne à voir ce qui relève selon nous d'une tentative de micro-improvisaton initiée par la consultante que la conseillère ne reprend pas à son compte et qui meurt en quelque sorte dans l'œuf. À ce stade d'un entretien mené de façon très directive, la consultante s'est assez peu exprimé : ses interventions sont brèves et peu élaborées. En C29, la conseillère aborde le thème des obstacles au projet, sous la forme d'une question, à laquelle la consultante répond par une reformulation en écho (D29: «des obstacles »), ce qui lui vaut une relance visant à complémenter la question posée préalablement $(\mathrm{C} 30$ : «à court, à moyen terme... »). À l'image de relances antérieures non reproduites ici suggérant un certain désarroi de D. (C10: «donc, par rapport à votre projet professionnel, ces expériences professionnelles vous ont apporté quoi ?», D10 : «Euh... Pardon ??? »), cette relance reste sans effet (D30 : «euh »). À ce moment, la conseillère reformule sa question plus explicitement (C31), ce qui entraîne une réponse dénuée d'ambiguïté : l'obstacle à son projet, «c'est ma petite fille...». Malgré toute sa richesse, attestée par les précautions oratoires qui accompagnent un discours nous apparaissant ici authentique et intime (« ça je le dirais pas si j'ai un entretien professionnel») et par le (relativement) long développement consacré à la thématique de la relation éducative (« elle a besoin de la présence de sa mère, d'une attention soutenue, qu'on l'éveille, qu'on joue avec elle et tout») qui vise à justifier que la petite fille puisse constituer un obstacle en soi, cette réponse n'est pas reprise dans l'intervention qui suit. À ce moment précis, de très nombreuses interventions sont imaginables de la part de la professionnelle du conseil : une centration sur l'arrivée de la petite fille dans la vie de sa maman, les ressources auxquelles elle pourrait faire appel pour suppléer sa présence, les difficultés qu'elle anticipe déjà lorsqu'elle dit «à un certain moment ce sera un peu dur »... Mais elle choisit ici de poursuivre sur la voie des obstacles pour en faire évoquer d'autres (C31). Cette intervention, vraisemblablement motivée par la volonté de rester fidèle au guide de questionnement, vient ici fermer une porte qui venait peut-être tout juste de s'entrouvrir. De dialogue improvisé donc il n'y aura pas. La conseillère n'exploitera pas ce qu'elle commençait tout juste à recueillir. La consultante qui essaiera un peu plus loin (sans plus de succès) de revenir à sa fille n'aura pas non plus la possibilité d'orienter l'entretien vers une voie non planifiée par son interlocutrice, bien qu'elle soit manifestement centrale pour elle.

On pourrait avancer que cet entretien met moins ses deux protagonistes aux prises avec l'improvisation qu'il ne les conduit à développer deux stratégies distinctes pour ne pas y avoir recours : par ses questions directives, la conseillère restreint l'espace de parole au point de réduire l'autre, sinon au silence, à des réponses factuelles qui ne l'impliquent guère. $\mathrm{Si}$, dans cet extrait, il est possible de parler de relatif insuccès de l'entretien, c'est bien parce que la conseillère, en s'interdisant tout écart par rapport à sa «feuille de route », fige les asymétries initiales et se prive ainsi de tout moyen de tirer parti de ce qu'une relation vive ouvre comme potentialités créatrices ; captive, moins de son rôle sans doute que de l'image 
qu'elle s'en fait, elle «sur-ritualise » l'échange au point de l'appauvrir, en écartant tout contenu qui apparaît ne pas répondre alors à ses attentes.

\section{6.- Conclusion}

Partant de l'idée que l'improvisation prend place dans de nombreux cours d'action, dialogués ou non, nous avions le projet de montrer en quoi certaines séquences extraites de notre corpus d'entretiens de conseil en orientation s'apparentaient à une véritable improvisation dialoguée. Plus précisément, nous supposions : 1) qu'en raison de l'asymétrie des rôles et des places conversationnelles dans cet entretien, les tentatives de ruptures seraient davantage le fait des consultants ; 2) que cette asymétrie se traduirait sur le plan discursif par un contraste entre un discours incertain du consultant et des interventions précises et finalisées du conseiller, contraste s'estompant cependant une fois ouverte la séquence d'improvisation dialoguée, sous l'effet de l'ajustement discursif du conseiller ; 3) que ces conduites d'ajustement donneraient lieu à des variations interindividuelles.

$\mathrm{Au}$ regard de ces attendus, reprenons ce que l'analyse des extraits d'entretiens de conseil nous a appris sur les formes de présence de l'improvisation :

1. Elle est liée au fait qu'en s'engageant dans la conversation, les interlocuteurs en présence ne savent pas vraiment à l'avance comment ils vont y interagir, ni précisément ce que chacun va y faire, ce qui les «condamne » à devoir gérer leurs actions respectives en situation, en fonction des exigences de l'instant présent. Si les consultants se montrent particulièrement peu préparés à l'exercice, au point de méconnaître le contrat de communication propre à cet entretien (Mme A. dans l'extrait 1), les conseillers sont, quant à eux, placés dans la position ambiguë de devoir mener un jeu qu'ils ne sauraient maîtriser complètement, puisque le déroulement effectif de l'entretien, tout comme son issue, reste fonction de ce que l'interaction aura produit à chaque étape ;

2. Elle est inaugurée par une rupture thématique et/ou conversationnelle toujours alimentée par le consultant. Surgissant dans le dialogue à la faveur de révélations, petites ou grandes, faisant appel à l'expression authentique d'une forme de récit de soi, les microimprovisations des consultants semblent s'adresser au moins autant à eux-mêmes qu'à leur destinataire. Systématiquement décalées par rapport au(x) tour(s) précèdent(s), elles marquent une rupture dans le cours «normal » de l'entretien en y introduisant d'autres contenus et/ou d'autres modes énonciatifs que ceux attendus dans le protocole ou en tout cas dans le moment présent, ouvrant dès lors une voie incertaine. On notera que dans les trois extraits présentés, elles surviennent avec la convocation de la sphère familiale sphère pertinente, mais non centrale dans l'entretien de conseil en orientation - à travers la question de la filiation ;

3. Elle engendre, du fait de son rattachement thématique très personnel et de sa dimension souvent réflexive, une mise en mots particulière qui, le plus souvent, a pour effet de démarquer le discours propre aux micro-improvisations des précédentes interventions du consultant. Avec des propositions encore plus hachées, s'exprimant sous forme de bribes accompagnées de nombreuses marques de modalisations exprimant le doute, cette mise en mots s'avère très difficile dans le cas B. (extrait 3), présentant toutes les caractéristiques du discours associé à l'intime (Pop, 2007). Sous la forme d'une assez longue bouffée tranchant avec les brèves réponses ou manifestations d'incompréhension précédentes, elle se révèle libératrice dans le cas de D. (extrait 5) tandis qu'elle apparaît particulièrement déterminée dans le cas de Mme A. qui convoque magistralement les première et troisième personnes, le passé et le présent pour livrer ce qu'elle a à dire (extrait 2) ;

4. Elle infléchit le discours des conseillers. Plutôt caractérisé par l'usage du questionnement et de la relance dans un cadre assez peu directif, mais néanmoins non dénué de 
structuration thématique, ce discours qui contraste avec celui du consultant dans le cadre habituel de l'entretien se met à s'en rapprocher structurellement lorsque la conseillère accepte d'entrer dans l'univers de ce qu'initie le consultant (extraits 2 et 3 ). Dans le cas le plus extrême (extrait 2), ajustant ses interventions au plus près des micro-improvisations de son partenaire, elle le suit alors pas à pas dans ses prises de parole, au moyen d'un usage abondant d'hétéro-reformulations, complétant ou reprenant en écho chacune de ses propositions. Son registre sémantique se situe dans le prolongement de celui du consultant, le temps d'une parenthèse au cours de laquelle elle modifie son objectif initial. Sans l'avoir perdu de vue pour autant, elle y revient dès lors qu'elle juge le registre thématique en cours déplacé par rapport au cadre de l'entretien de conseil en orientation. Elle s'emploie alors à clôturer la séquence, par le biais d'une rupture conversationnelle visant à réorienter l'entretien vers son cours « normal ».

5. Il y a cependant sur ce point d'importantes différences entre les professionnelles de l'orientation : si l'ajustement est maximal pour la conseillère en interaction avec Mme A., il est moins remarquable dans sa forme pour celle qui s'entretient avec B. - dont l'usage de questions à tonalité intrusive contraste avec les bribes énoncées par son partenaire - et totalement absent du discours de la conseillère interagissant avec D. qui, loin de suspendre le déroulement de son questionnement pour un temps - celui nécessaire à l'écoute des éléments personnels évoqués dans l'instant -, poursuit imperturbablement sa trame propre. De ce point de vue et par contraste, on mesure combien l'ajustement à l'autre dans le cadre d'une séquence dialoguée improvisée exige d'attention, de réactivité, de capacité à faire face pour être en mesure de décider, à chaque tour de parole, des significations à attribuer à l'action langagière de l'autre et des enchaînements conversationnels à accomplir soi-même, notamment lorsque l'on est un jeune praticien pas nécessairement expérimenté en matière d'entretien de conseil centré sur l'écoute comme c'est le cas pour notre échantillon.

6. Dès lors, et au vu des effets bénéfiques qu'un déplacement thématique de l'échange induit manifestement aux dires de B. (extrait 4), il nous semble que le succès (ou l'insuccès) de l'entretien dépend en partie de la capacité du conseiller à accueillir cette parole et à en rendre possible l'élaboration conjointe, selon des modalités qui restent à réinventer dans chaque situation singulière.

Notre ambition était de soumettre à l'analyse l'activité ordinaire d'entretien de conseil en orientation en nous focalisant sur la part improvisée de l'activité et en prenant appui sur une analyse discursive. Au terme de cette présentation, quelques éléments peuvent être discutés.

En premier lieu, la notion d'improvisation en elle-même est souvent dévalorisée dans les milieux de formation et de travail. Nous voudrions insister sur le fait que, signifiant littéralement «sans voir avant» (improvisus: in, privatif et providere : voir avant), l'improvisation renvoie moins à un manque de préparation, qu'à une absence de planification. En effet, on ne saurait envisager la possibilité d'improviser dans le champ professionnel sans l'existence a minima d'un canevas d'action, toute action improvisée s'effectuant en fonction des capacités (déjà là) du sujet à utiliser les possibilités offertes par la situation dans laquelle il se trouve (Azéma \& Leblanc, 2011 ; Perrenoud, 1994). Vue sous cet angle, l'improvisation au sens de « gestes d'ajustement» ou de «micro-improvisations » décrites par Blucheton (2009), est intéressante à observer chez de jeunes professionnels soucieux de mieux analyser leurs pratiques. Car apprendre à agir adéquatement face aux imprévus, devenir capable de construire une réponse originale à une situation inédite est un enjeu capital de la formation pour bien des métiers, y compris ceux qui s'exercent principalement au moyen de la parole. À défaut, l'urgence nécessairement rencontrée un moment donné dans le travail a de fortes probabilités d'accroître la possibilité d'une «improvisation réglée » qui fonctionnerait comme un révélateur de l'habitus, suscitant des réponses sans qu'il y ait eu ni délibération intérieure consciente ni recours à des savoirs explicites (Bourdieu, 1980 ; Perrenoud, 1999). Pour mieux s'en distancier, il importe alors 
de soumettre à l'analyse des situations de travail marquées par des moments de gestion de l'imprévu: interpréter les enjeux de telles situations, repérer quels en sont les signes précurseurs, analyser comment les réponses sont activées, identifier d'autres réponses alternatives, voici quelques pistes d'action utiles pour la formation de praticiens.

Deuxièmement, il nous faut tout de même relativiser la part improvisée de l'entretien de conseil. Si l'on ne conteste pas le fait qu'elle soit partie intégrante de l'activité de conseil, notre micro-analyse a cependant montré que dans la pratique, ce n'est jamais l'orientation générale de l'entretien qui est improvisée, puisque le conseiller poursuit un objectif, en général fonction de la finalité du type d'entretien concerné, et de l'attente qu'exprime le consultant. Deux choses en revanche sont toujours au moins en partie improvisées : la gestion de la relation durant l'échange d'une part, et le «chemin » par lequel l'objectif de l'entretien sera atteint (s'il l'est), le conseiller étant toujours dans l'ignorance de ce que sera le tour d'après, tant du point de vue de la relation que du point de vue de la progression thématique. De ce point de vue, mettre en exergue le caractère circonscrit de cette part aléatoire est tout l'intérêt du choix de la micro-improvisation comme unité d'analyse.

Troisièmement, la méthode utilisée, une micro-analyse énonciative et interactionnelle de cas singuliers prélevés dans un vaste corpus échappe, certes, à toute tentative de généralisation (Chabrol \& Olry-Louis, 2007 ; Dumazière \& Dubar, 2009). Mais elle offre en contrepartie la possibilité de donner à voir la manière dont s'accomplit concrètement l'entretien de conseil en orientation et, ce faisant, d'envisager les dimensions créatives de «l'agir » à travers la question : comment les cadres interactionnels propices à l'improvisation émergent-ils au niveau intersubjectif (Bachir-Loopuyt, Canonne, Saint-Germier, \& Turquier, 2010)? Constituant de véritables incursions dans la matérialité de la mise en mots, de telles démarches d'analyse qualitative prennent une dimension ethnographique lorsqu'elles font porter la description sur une situation professionnelle. Dès lors elles sont capables de renseigner sur le métier (Olry-Louis, Soidet, \& Duveau, 2013), sur le rapport au métier (Olry-Louis \& Olry, 2011) ou encore sur la dimension sociale du langage dans les interactions professionnelles de métier (Boutet \& Gardin, 2001 ; Olry-Louis \& Cartron, 2009 ; Olry-Louis \& Tamburini, 2011). Mises en oeuvre à un niveau très fin, elles font souvent poindre, comme dans le cas présent, des différences inter-individuelles suggérant une perfectibilité que l'on pourrait chercher à développer chez les professionnels par le truchement d'analyses différées des pratiques. En effet, les métiers comme le conseil en orientation articulent étroitement un agir verbal et un agir de travail pourtant bien distincts : contribuant à la réalisation du travail, le premier renvoie d'abord à un système de contraintes incorporées mettant en jeu l'énonciation, alors que le second s'inscrit dans une histoire sédimentée propre au collectif de travail et à l'institution. Et comme le pointe Boutet (2005), si les situations sociales jouent sur les pratiques langagières en prédéterminant pour une part leur forme - avec de fortes contraintes exercées sur le format de l'interaction, les dialogues analysés ici restant fortement structurés par des institutions -, d'un autre côté, les pratiques langagières peuvent aussi agir sur les situations sociales en les faisant évoluer. Aussi, pour ce secteur professionnel où les conditions d'emploi sont souvent difficiles et où l'on fait volontiers appel à un personnel jeune et peu expérimenté, ce n'est pas la seule activité de travail qui doit faire l'objet d'une formation : il faut aussi examiner les activités langagières mises en œuvre au travail.

De ce point de vue, cette contribution a permis selon nous non seulement d'étayer les analyses conversationnelles pour ce domaine professionnel, mais aussi d'interroger la diversité des pratiques langagières dans la limite des trois entretiens choisis, objectif qui nécessiterait bien sûr une poursuite du travail descriptif et comparatif amorcé. Dans cette perspective, on pourrait chercher à donner à la comparaison un caractère plus systématique à partir de critères bien précis portant sur un grand nombre d'extraits, ou en accordant aux cas examinés un statut prototypique en définissant soigneusement la nature des prototypes choisis. 


\section{BIBLIOGRAPHIE}

Alvesson, M., \& Karreman, D. (2000). Varieties of discourse: On the study of organisations through discourse analysis. Human relations, 53 (9), 1125-1149.

Azéma, G., \& Leblanc, S. (2011). L’improvisation, objet paradoxal et praxis incontournable du travail ordinaire de l'enseignant. Colloque international de l'INEP, 16-18 mars 2011. [Consulté de http://www.inrp.fr/archives/colloques/travail-enseignant/contrib/38.htm]

Bachir-Loopuyt, T., Canonne, C., Saint-Germier, P., \& Turquier, B. (Eds.) (2010). Improvisation : usages et transferts d'une catégorie. Tracés, Revue de sciences humaines, 18 [consulté de : http://traces.revue.org/index4499.html].

Béguin, P., \& Clot, Y. (2005). L'action située dans le développement de l'activité. @ ctivités, 1(2), 2749.

Bénony, H., \& Chahraoui, K. (1999). L'entretien clinique. Paris: Dunod.

Benvéniste, E. (1974). Problèmes de linguistique générale (volume 2). Paris: Gallimard.

Bernaud, J.-L. (2012). L'efficacité du bilan de compétences : pistes pour l'évolution des prestations. Éducation Permanente, 192, 55-69.

Blanchet, A., \& Gotman, A. (2006). L'enquête et ses méthodes : l'entretien. Paris: Nathan.

Blumer, H. (1969). Symbolic interactionnism. Englewoods Cliffs, N. J.: Prentice Hall.

Bourdieu, P. (1980). Le sens pratique. Paris: Édition de Minuit.

Boutet, J. (2005). Genre de discours et activité de travail. In L. Filliettaz, \& J.-P. Bronckart (Eds.), L'analyse des actions et des discours en situation de travail. Concepts, méthodes et applications (pp.19-35). Louvain-la-Neuve: Peeters.

Boutet, J., \& Gardin, B. (2001). Une linguistique du travail. In A. Borzeix \& B. Fraenkel (Eds), Langage et travail. Communication, action, cognition (pp. 89-112). Paris: CNRS éditions.

Bucheton, D. (Ed.) (2009). L'agir enseignant: des gestes professionnels ajustés. Toulouse: Octarès Éditions.

Camus, O. (2004). L'entretien de recrutement : contrat très particulier ou terrain modèle ? In M. Bromberg \& A. Trognon (Eds), Psychologie sociale et communication. (pp. 157-172). Paris: Dunod.

Chabrol, C. (2000). Contrats de communication et entretiens d'orientation, dispositif clinique ou psychosocial ? L'orientation scolaire et professionnelle, 29, 171-180.

Chabrol, C., \& Olry-Louis, I. (Eds.) (2007). Interactions communicatives et psychologie. Paris: Presses de la Sorbonne Nouvelle.

Conein, B., \& Jacopin, E. (1994). Action située et cognition. Le savoir en place. Sociologie du travail, 4, 475-500.

Dejours, C. (2009). Le travail vivant. Paris: Payot.

Delory-Momberger, C. (2007). La biographisation des parcours entre projet de soi et cadrage institutionnel. L'orientation Scolaire et Professionnelle, 36(1), 9-17.

Doublet, M.-H. (2006). Étude des interactions conseiller bénéficiaire dans le bilan de compétences (thèse de doctorat non publiée). Paris: CNAM.

Drew, P., \& Heritage, J. (Eds.). (1992). Talk and work. Cambridge: Cambridge University Press.

Dumazière, D., \& Dubar, C. (2009). Analyser les entretiens biographiques. L'exemple de récits d'insertion. Québec: Presses de l'université de Laval.

Egan, G. (1994). The Skilled Helper. Pacific Grove (Cal.): Brooks/Cole.

François, F. (1993). Pratiques de l'oral, dialogue, jeu et variation autour des figures de sens. Paris: Nathan.

Goffman, E. (1973). La présentation de soi dans la vie quotidienne. Paris: Édition de Minuit.

Grossen, M. (2001). La notion de contexte : quelle définition pour quelle psychologie ? Un essai de mise au point. In J.-P. Bernié (Ed.), Apprentissage, développement et significations (pp. 59-77). Bordeaux: Presses universitaires de Bordeaux.

Grossen, M., \& Salazar Orvig, A. (2006). Introduction : variété et hétérogénéité des entretiens cliniques. In M. Grossen, \& A. Salazar Orvig (Eds), L'entretien clinique en pratiques - Analyse 
des interactions verbales d'un genre hétérogène (pp. 9-28). Paris: Belin.

Grossen, M. \& Trognon, A. (2002). L'entretien clinique : une conversation ordinaire ? In J. Bernicot, M. Grossen, A. Trognon, \& M. Musiol (Eds), Pragmatique et psychologie (pp. 139-153). Nancy: P.U.N.

Guichard, J. (2008). Proposition d'un schéma d'entretien constructiviste de conseil en orientation (life designing counseling) pour des adolescents ou de jeunes adultes. L'Orientation Scolaire et Professionnelle, 37(3), 413-440.

Guichard, J., \& Huteau, M. (2007). Orientation et insertion professionnelle, 75 concepts clés. Paris: Dunod.

Gysbers, N.C., Heppner, M.J., \& Johnston, A. (1998). Career Counseling: Process, Issues, and Techniques. Needham Heights (MA.): Allyn \& Bacon.

Hansen, W-E. (2005). Mixed methods research designs in counseling psychology. Journal of Counseling Psychology, 52, 224-235.

Hanson, W.E., Creswell, J.W., Plano Clark, V.L., Petska, K.S., \& Creswell, J.D. (2005). Mixed method research design in counseling psychology. Journal of Counseling Psychology, 52,224-235.

Kerbrat-Orecchioni, C. (2005). Le discours en interaction. Paris : Armand Colin.

Kostulski, K., \& Prot, B (2004). L'activité conversationnelle d'un jury de Validation d'Acquis : analyse interlocutoire de la formation d'un concept potentiel ? Psychologie Française, 49(4), 425441 .

Labov, W., \& Fanshel, D. (1977). Therapeutic Discourse. London: Academic Press.

Lacaille, S. (2012). Les ingrédients actuels du bilan de compétence sont-ils solubles dans le cadre réglementaire des années 1990 ? Éducation Permanente, 192, 143-161.

Léontiev, A.N. (1974). The problem of activity in psychology. Soviet Psychology, 13, 14-33.

Lhotellier, A. (2000). L'acte de tenir conseil. L'Orientation Scolaire et Professionnelle, 29(1), 27-50.

Licoppe, C. (2008). Dans le « carré de l'activité »: perspectives internationales sur le travail et l'activité. Sociologie du travail, 50(3), 287-302.

Livingstone, E. (2008). Context and detail in studies of the witnessable social order: Puzzles, maps, checkers, and geometry. Journal of Pragmatics, 40, 840-862.

Manthei, R. (1997). Counselling: the Skills of Finding Solutions to Problems. Londres: Routledge.

Mayen, P. (2009). Entretien de validation. In J.-P. Boutinet (Ed.), L'ABC de la VAE (pp. 134-136). Toulouse: Ères.

Mead, G. H. (1934). Mind, self and society. Chicago: University of Chicago Press.

Ogien, A., \& Quéré, L. (2005). Le Vocabulaire de la sociologie de l'action. Paris: Ellipse.

Olry-Louis, I. (2013). Les interactions de conseil. In I. Olry-Louis, V. Guillon \& E. Loarer (Eds.), Psychologie du conseil en orientation (pp. 65-83). Bruxelles: de Boeck.

Olry-Louis, I., \& Brémond, C. (2010). La construction d'un cadre pour la confidence : le cas des entretiens de conseil en orientation. In M.-D. Popelard (Ed.). Les voix risquées de la confidence (pp. 29-53). Paris: Presses de la Sorbonne nouvelle.

Olry-Louis, I., Brémond, C., \& Pouliot, M. (2012). Confidence-sharing in the career counseling interview: emergence and repercussions. International Journal for Educational and Vocational Guidance, 12(1), 47-65.

Olry-Louis, I., \& Cartron, B. (2009). Négociation dans une agence publicitaire : l'analyse d'une réunion de travail à partir des actes de parole. Psychologie du Travail et des Organisations, 15(3), 271-296.

Olry-Louis, I., \& Grillo, E. (2014). Improviser en dialoguant. Le cas de l'entretien de conseil. In M.D. Popelard (Ed.). Improviser, une action dialogique (pp. 43-59). Rennes: Presses Universitaires de Rennes.

Olry-Louis, I., \& Olry, P. (2011). Transmettre la relation de service par l'interaction tutorale : le cas des facteurs. Psychologie du Travail et des Organisations, 17(3), 269-289.

Olry-Louis, I., Soidet, I., \& Duveau, V. (2013). Activités dialogiques de reformulation dans une liste de discussion professionnelle. In C. van de Leemput, C. Chauvin, \& C. Helemans (Eds.), Activités humaines, technologies et bien-être, actes du 7ème colloque de psychologie ergonomique Épique 
2013 (pp. 389-397). Paris: Arpège Science Publishing.

Olry-Louis, I., \& Tamburini, L. (2011). La parole au travail a-t-elle un genre ? Dialogues hôtesse de l'air/steward en résolution de problème. Psychologie du Travail et des Organisations, 17(4), 362 381.

Perrenoud, P. (1994). La pratique pédagogique entre improvisation réglée et bricolage. In $\mathrm{Ph}$. Perrenoud (ed.). La formation des enseignants entre théorie et pratique (pp. 21-44). Paris: L'Harmattan.

Perrenoud, P. (1999). Gestion de l'imprévu, analyse de l'action et construction de compétences. Éducation Permanente, 140(3), 123-144.

Plantin C. (2003). Structures verbales de l'émotion parlée ou de la parole émue. In Colletta, J.-M. \& Tcherkassof, A. (Ed.), Les émotions. Cognition, langage et développement (pp. 97-130), Liège: Mardaga.

Pop, L. (2007). La confidence, genres, marqueurs, stratégies et niveaux de textualisation. In C. Kerbrat-Orecchioni, \& V. Traverso (Eds.), La confidence et le dévoilement de soi dans l'interaction (pp. 407-426). Tübingen: Niemeyer.

Popelard, M.-D. (Ed.) (2014). Improviser, une action dialogique. Rennes: Presses Universitaires de Rennes.

Rogers, C. R. (1951). Client-Centered Therapy. Boston: Houghton Mifflin Compagny.

Sacks, H. (1992). Lectures on Conversation, Volumes I and II. Oxford: Blackwell.

Sacks, H., Schegloff, E. A., \& Jefferson, G. (1974). A Simplest Systematics for the Organisation of Turn-Taking for Conversation. Language, 50, 696-735.

Savickas. M. (2011). Career Counseling. Washington, DC: American Psychological Association.

Schegloff, E.A. (1987) Analyzing single episodes of interaction: an exercise in conversation analysis. Social Psychology Quarterly, 50, 101-14.

Schilling, J. (2006). On the pragmatics of qualitative assessment. European Journal of Psychological Assessment, 22, 28-35.

Suchman, L. (1987). Plans and situated actions; the problem of human machine interaction. Cambridge: Cambridge University Press.

Trognon, A. (1999). Éléments d'analyse interlocutoire. In M. Gilly, J. P. Roux, \& A. Trognon (Eds.), Apprendre dans l'interaction (pp. 69-94). Nancy: Presses Universitaires de Nancy.

Trognon, A. (2002). Speech Acts and the Logic of Mutual Understanding. In D. Vanderveken, \& S. Kubo (Eds.). Essays in Speech Acts Theory (pp. 121-133). Amsterdam: John Benjamins ans sons.

Vion, R. (1992). La communication verbale. Analyse des interactions. Paris: Hachette.

Vygotski, L.S. (1997). Pensée et langage. Paris: La dispute.

Zarka, J. (2000). Conseils et limites : limites du conseil au-delà des limites. L'Orientation Scolaire et Professionnelle, 29, 141-169.

\section{RESUME}

Défendant l'intérêt qu'il y aurait à considérer les interactions ordinaires de conseil en orientation comme une activité qu'il convient d'analyser en référence aux théories de l'activité, à l'action située et à l'analyse conversationnelle, nous soutenons une position qui peut être résumée ainsi : 1) une part d'improvisation est partie intégrante de l'activité de conseil, 2) cette part d'improvisation (ou micro-improvisations) procède des caractéristiques mêmes de la situation, et notamment, de l'asymétrie qu'elle instaure entre les protagonistes, le bénéficiaire ayant à fournir une matière permettant au conseiller d'accomplir interactionnellement ses gestes professionnels, 3) s'il est permis d'affirmer que les deux partenaires improvisent compte tenu de cette asymétrie, ils ne le font pas de la même manière, le bénéficiaire n'ayant pas toujours une vision claire de ce qu'on attend vraiment de lui et le conseiller ne pouvant pas 
nécessairement prévoir l'effet de ses interventions, 4) dès lors, le « succès » de l'entretien dépendrait de la complémentarité qu'ils parviendraient (ou pas) à réaliser dialogiquement, via leurs interventions respectives dans un procès relevant alors d'une véritable improvisation dialoguée. Il s'agit donc d'aborder le conseil en orientation comme une activité conjointe s'élaborant dans l'iciet-maintenant d'une interaction vive et, à partir de l'analyse des échanges langagiers, de mettre au jour la manière dont les protagonistes parviennent conjointement à faire progresser l'entretien. Pour étayer empiriquement cette position, nous soumettons à une analyse de discours quelques extraits d'un corpus d'entretiens menés par de jeunes praticiens du conseil en orientation.

\section{MOTS-CLEFS}

activité, analyse conversationnelle, interactions dialogiques, entretien de conseil en orientation, improvisation

\section{REFERENCEMENT}

Olry-Louis, I. (2015). Activité dialogique et micro-improvisations en entretien de conseil en orientation. Activités, 12(1), 3-23. http://www .activites.org/v12n1/v12n1.pdf

Article soumis le 30/03/2014, accepté le 26/01/2015 